\title{
The dimension of remainders of rim-compact spaces
}

\author{
by
}

\author{
J. M. A arts and E. Coplakova (Delft)
}

\begin{abstract}
Answering a question of Isbell we show that there exists a rim-compact space $X$ such that every compactification $Y$ of $X$ has $\operatorname{dim}(Y \backslash X) \geq 1$.

It is known that a space $X$ is rim-compact if and only if $X$ has a compactification $Y$ such that $Y \backslash X$ is zero-dimensionally embedded in $Y$, i.e. $Y$ has a base for the open sets whose boundaries are disjoint from $Y \backslash X$. Note that this implies that $\operatorname{ind}(Y \backslash X) \leq 0$.

On the other hand, if there is a compactification $Y$ of the space $X$ such that ind $(Y \backslash X) \leq 0$ then $X$ need not be rim-compact; an example was given by Smirnov in [1958].

If $X$ is Lindelöf at infinity, i.e. each compact subset of $X$ is contained in a compact set with a countable base for its neighbourhoods, then $X$ is rimcompact if and only if it has a compactification $Y$ such that $\operatorname{ind}(Y \backslash X) \leq 0$; such a compactification then also has $\operatorname{dim}(Y \backslash X) \leq 0$. This suggests the question whether every rim-compact space $X$ has a compactification $Y$ such that $\operatorname{dim}(Y \backslash X) \leq 0$ (see Isbell [1964] and Aarts and Nishiura [1993]; see also Diamond, Hatzenbuhler and Mattson [1988] for related problems). We shall show that the answer is negative. We construct a strongly zero-dimensional space $X$ such that for every compactification $Y$ of $X$ we have $\operatorname{dim}(Y \backslash X) \geq 1$. Our space $X$ will be such that $\beta X \backslash X$ is metrizable, zero-dimensional but not strongly zero-dimensional. Originally, the remainder was Roy's space $\Delta$; however, by using Kulesza's space we were able to obtain an example of the smallest possible weight $\omega_{1}$.
\end{abstract}

Convention. We identify an ordinal with its set of predecessors so that for example $\omega_{1}+1=\omega_{1} \cup\left\{\omega_{1}\right\}$. All ordinals under consideration carry the order topology.

1991 Mathematics Subject Classification: Primary 54F45; Secondary 54D35. 
The construction. Let $K$ be Kulesza's space, i.e. $K$ is a (completely) metrizable subspace of $\omega_{1}^{\omega}$ of weight $w(K)=\omega_{1}$ which is dense in $\omega_{1}^{\omega}$ and satisfies ind $K=0$ and Ind $K=1$ (see Kulesza [1990]).

Consider now

$$
Z=\left(\omega_{1}+1\right)^{\omega+1}=\left(\omega_{1}+1\right)^{\omega} \times\left(\omega_{1}+1\right)
$$

and let

$$
X=Z \backslash\left(K \times\left\{\omega_{1}\right\}\right) .
$$

Then $\left(\omega_{1}+1\right)^{\omega} \times \omega_{1} \subseteq X \subseteq Z$. As $\left(\omega_{1}+1\right)^{\omega}$ is compact we may conclude that $Z=\left(\omega_{1}+1\right)^{\omega} \times\left(\omega_{1}+1\right)$ is the Cech-Stone compactification of $\left(\omega_{1}+1\right)^{\omega} \times \omega_{1}$ (see Engelking [1989, Problem 3.12.20(c)]). Hence $\beta X=Z$ as well. Note that $\beta X$ is a product of compact zero-dimensional spaces hence $\beta X$ is also compact and zero-dimensional and therefore strongly zero-dimensional. It follows that $X$ itself is strongly zero-dimensional, hence zero-dimensional and a fortiori rim-compact. It is also easily seen that $w(X)=\omega_{1}$. It remains to show that $\operatorname{dim}(\alpha X \backslash X) \geq 1$ for every compactification $\alpha X$ of $X$.

Let $\alpha X$ be a compactification of $X$. Consider

$$
f: \beta X \rightarrow \alpha X,
$$

the extension of the natural embedding $\operatorname{id}_{X}: X \rightarrow \alpha X$ over $\beta X$. As $\beta X$ and $\alpha X$ are compact, the mapping $f$ is perfect. Now,

$$
\beta X \backslash X=f^{-1}[\alpha X \backslash X]
$$

so $f\left\lceil\beta X \backslash X\right.$ is also perfect. But $\beta X \backslash X=K \times\left\{\omega_{1}\right\}$ and $K$ is metrizable. To finish our argument we need the following theorem, due to Morita and Nagami (see Engelking [1989]).

TheOREM. If $f: X \rightarrow Y$ is a closed mapping of a metrizable space $X$ to a metrizable space $Y$ and for every $y \in Y$, Ind $f^{-1}[\{y\}] \leq k$ for $k \geq 0$, then Ind $X \leq \operatorname{Ind} Y+k$.

Now, $K \times\left\{\omega_{1}\right\}$ and $\alpha X \backslash X$ are metrizable, the mapping $f: K \times\left\{\omega_{1}\right\} \rightarrow$ $\alpha X \backslash X$ is perfect and for each $x \in \alpha X \backslash X$ the fiber $f^{-1}[\{x\}]$ is compact and zero-dimensional, hence strongly zero-dimensional. Note that this means that $\operatorname{Ind}\left(f^{-1}[\{x\}]\right) \leq 0$ for each $x \in \alpha X \backslash X$, so by the theorem

$$
\operatorname{Ind}\left(K \times\left\{\omega_{1}\right\}\right) \leq \operatorname{Ind}(\alpha X \backslash X)+0,
$$

hence

$$
\operatorname{dim}(\alpha X \backslash X)=\operatorname{Ind}(\alpha X \backslash X) \geq \operatorname{Ind}\left(K \times\left\{\omega_{1}\right\}\right)=1 .
$$

\section{References}

J. M. Aarts and T. Nishiura [1993], Dimension and Extensions, Elsevier, Amsterdam. 
B. Diamond, J. Hatzenbuhler and D. Mattson [1988], On when a 0-space is rimcompact, Topology Proc. 13, 189-202.

R. Engelking [1989], General Topology, revised and completed edition, Sigma Ser. Pure Math. 6, Heldermann, Berlin.

J. R. Is bell [1964], Uniform Spaces, Math. Surveys 12, Amer. Math. Soc., Providence, R.I.

J. Kulesza [1990], An example in the dimension theory of metrizable spaces, Topology Appl. 35, 109-120.

Yu. M. Smirnov [1958], An example of a completely regular space with zero-dimensional Čech remainder, not having the property of semibicompactness, Dokl. Akad. Nauk SSSR 120, 1204-1206 (in Russian).

FACULTY OF TECHNICAL MATHEMATICS AND INFORMATICS

TU DELFT

POSTBUS 5031

2600 GA DELFT, THE NETHERLANDS

E-mail: AARTS@DUTIAW3.TWI.TUDELFT.NL

EVA@DUTIAW3.TWI.TUDELFT.NL

Received 14 May 1993

\section{FUNDAMENTA MATHEMATICAE 143.3}

(c) Copyright by Instytut Matematyczny PAN, Warszawa 1993

PRINTED IN POLAND 